\title{
Post-Traumatic Diaphragmatic Hernia -Delayed Presentation with Complications
}

\author{
Revin $^{1}$, Rohini Avantsa² ${ }^{2}$ U. Shrikrishna ${ }^{3}$ \\ ${ }^{1}$ Department of Radiodiagnosis, KS Hegde Medical Academy, Mangalore, Karnataka, India. \\ ${ }^{2}$ Department of Radiodiagnosis, KS Hegde Medical Academy, Mangalore, Karnataka, India. \\ ${ }^{3}$ Department of Radiodiagnosis, KS Hegde Medical Academy, Mangalore, Karnataka, India.
}

\section{INTRODUCTION}

Diaphragmatic hernia is due to a defect in the diaphragm which can be either congenital or acquired, leading to herniation of abdominal contents into the thoracic cavity. The most common cause of acquired diaphragmatic hernias is trauma. Traumatic diaphragmatic injuries that are acquired are uncommon and are missed frequently due to non-specific features and initial small size of hernia. Blunt or penetrating thoracic or abdominal injury can result in traumatic diaphragmatic hernia. Post-traumatic diaphragmatic hernias are more common on the left side compared to the right side because of congenitally strong right hemidiaphragm and protection by the liver. $0.8-3.6 \%$ of blunt trauma patients present with right-sided hernia. Proper diagnosis of both benign and life-threatening manifestations of diaphragmatic hernias is very important for further management. $[1,2,3,4]$ Diaphragmatic hernias are uncommon, and at times are incidental. Diaphragmatic hernias are of two types, congenital and traumatic. Traumatic diaphragmatic hernias are rare. We are presenting a case of 72 years old male with incidentally detected right-sided diaphragmatic hernia with classical signs which helped in diagnosing the condition.

\section{PRESENTATION OF CASE}

A 72-year-old male was apparently normal 15 days back when he developed multiple episodes [2-3 episodes per day] of vomiting which were non-projectile, moderate, non-bilious, not blood-stained and containing food particles. The patient also complained of breathlessness and backache for 15 days which was insidious in onset, and progressed gradually. Pain and breathlessness increase on activity and reduce on rest. The patient gives a history of trauma 12 years back resulting in fractures of T5, T12 vertebrae. He was managed conservatively with rest for the same. Mild tenderness was noted in the upper abdomen on palpation. On auscultation, no breath sounds were heard in the right hemithorax. Preoperative chest Radiograph revealed opacity in the right hemithorax and referred to the radiology department for further evaluation. PA and lateral chest radiograph, barium meal follow-through, CT thorax, and abdomen were performed. The patient was further investigated with upper GI endoscopy.
Corresponding Author: Dr. Revin,

H. No. 1612, Sector-10A,

Urban Estate,

Opp. Meenakshi Public School, Gurgaon-122001, Haryana, India.

E-mail:dr.revin@gmail.com

DOI: $10.14260 / \mathrm{jemds} / 2020 / 150$

Financial or Other Competing Interests: None.

How to Cite This Article:

Revin, Avantsa R, Shrikrishna U. Posttraumatic diaphragmatic hernia- delayed presentation with complications. J. Evolution Med. Dent. Sci. 2020;9(09):692696, DOI: $10.14260 /$ jemds/2020/150

Submission 21-12-2019,

Peer Review 02-02-2020

Acceptance 11-02-2020,

Published 02-03-2020.

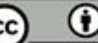




\section{RESULTS}

Chest radiograph revealed multiple air-fluid levels in right hemithorax with indistinct diaphragm, mild mediastinal shift, continuation of bowel loops from peritoneum to thoracic cavity. A suspicious diagnosis of diaphragmatic hernia was made on chest radiography. CT scan was performed for further evaluation. Contrast-enhanced CT Thorax showed absent right hemidiaphragm for an anterior-posterior diameter of $17 \mathrm{cms}$. few anterior fibers of diaphragm are seen which showed mild thickening. There is herniation of intra and retroperitoneal contents into the right hemithorax causing passive collapse of right lung middle and lower lobes which are displaced towards mediastinum. Pulmonary artery and veins are of normal caliber. Main bronchi and the lobar bronchi appear patent.

\section{Following Abdominal Structures Herniated in to the} Right Hemithorax

1. Bowel Loops: Distal stomach, gastro-duodenal junction, C-loop of duodenum, duodenojejunal junction, entire jejunum, ileum, ileocecal junction, ascending colon, hepatic flexure, proximal and middle transverse colon. The distal transverse colon, descending colon, sigmoid colon, and splenic flexure are seen in the abdominal cavity. There is an inflammatory wall thickening of the stomach with mucosal hyperenhancement and submucosal edema.

2. Solid Intraperitoneal Structures: Left lobe of the liver, gallbladder, head and uncinate process of the pancreas, Mesentery, omentum and vascular structures (Portal vein, distal splenic vein, SMV, mesenteric vessels, celiac artery, and its branches).

\section{Radiological Findings/ Signs Observed} Radiograph/MDCT Which Confirmed the Diagnosis

- Air-fluid levels in the thoracic cavity [Radiograph].

- Elevated hemidiaphragm [Radiograph, CT].

- Diaphragm not visualized [CT and Radiograph].

- Deviation of the mediastinum towards the non-affected side [Radiograph, CT].

- Protrusion of abdominal organs or peritoneal fat into the pleural space [Radiograph, CT].

- Loss of diaphragmatic continuity with thickening of the free edge [CT].

- Dangling diaphragm sign-Thickened torn, comma-shaped curved edge [CT].

- Collar sign (or hourglass sign) -secondary to compression of herniated structure at the site of rupture [CT].

- Dependent viscera sign-herniated abdominal contents in direct contact with posterior thoracic wall [CT].

- Hump sign-due to hepatic herniation [CT].

- Vertebral fractures [Radiograph, CT].

Upper GI endoscopy was done which showed Antral gastritis with bile reflux and duodenitis. Even after explaining the possible complications of diaphragmatic hernia to the patient, he was not willing for surgical intervention and was subsequently discharged.
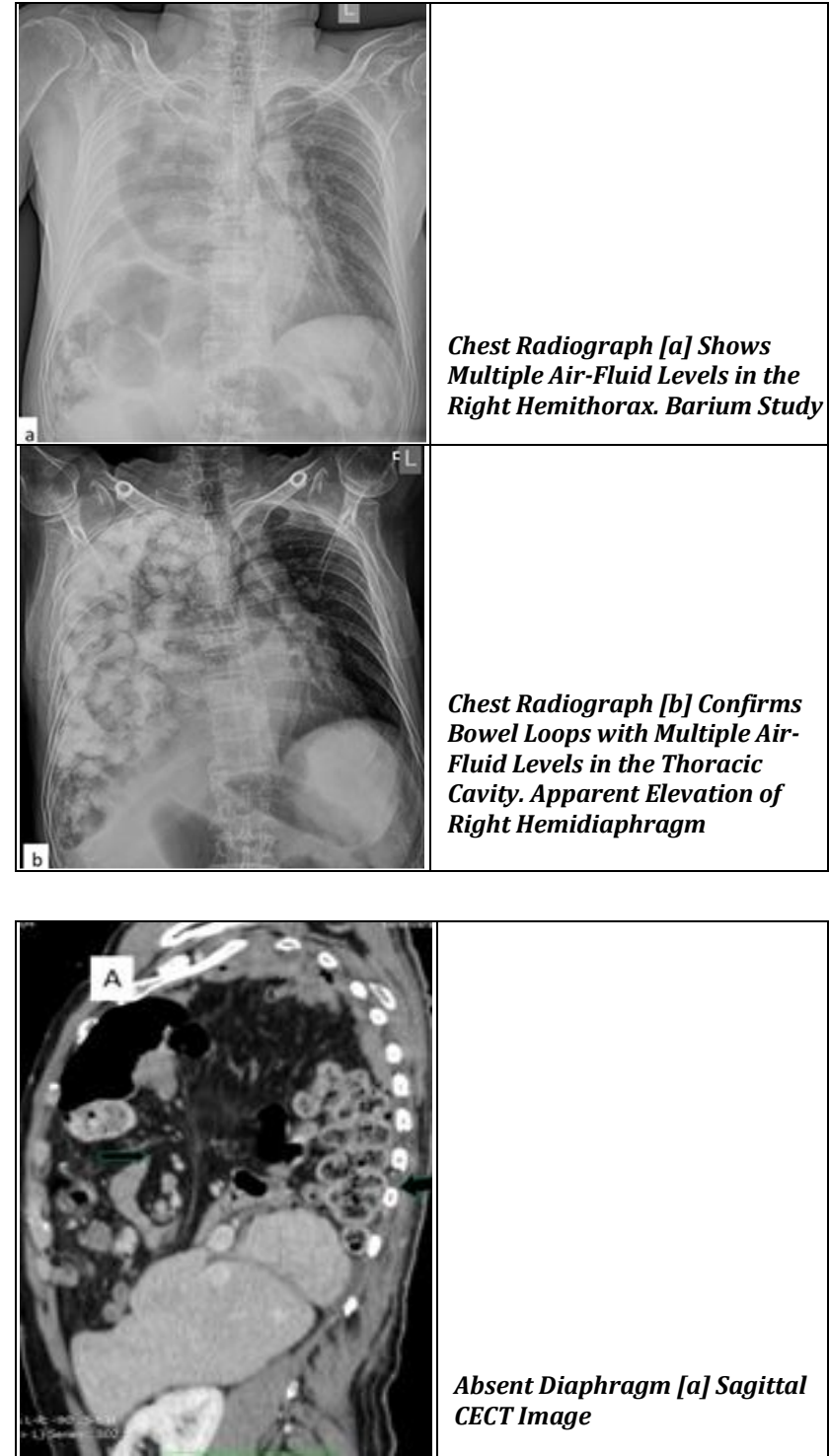

Absent Diaphragm [a] Sagitta CECT Image

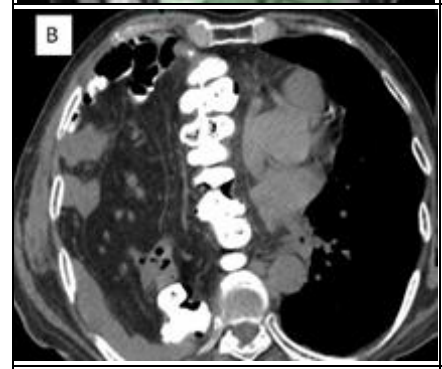

Absent Diaphragm [b] Axial CECT Image

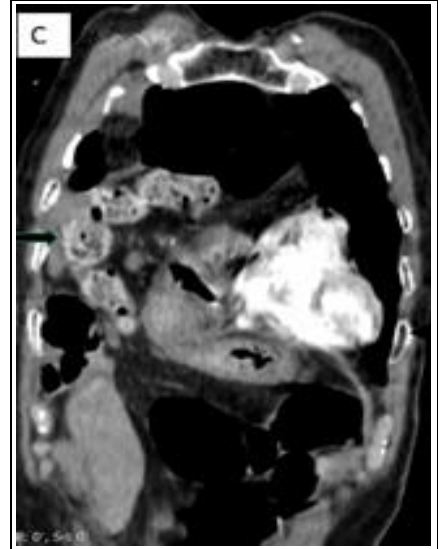

Absent Diaphragm [c] Coronal CECT Image 

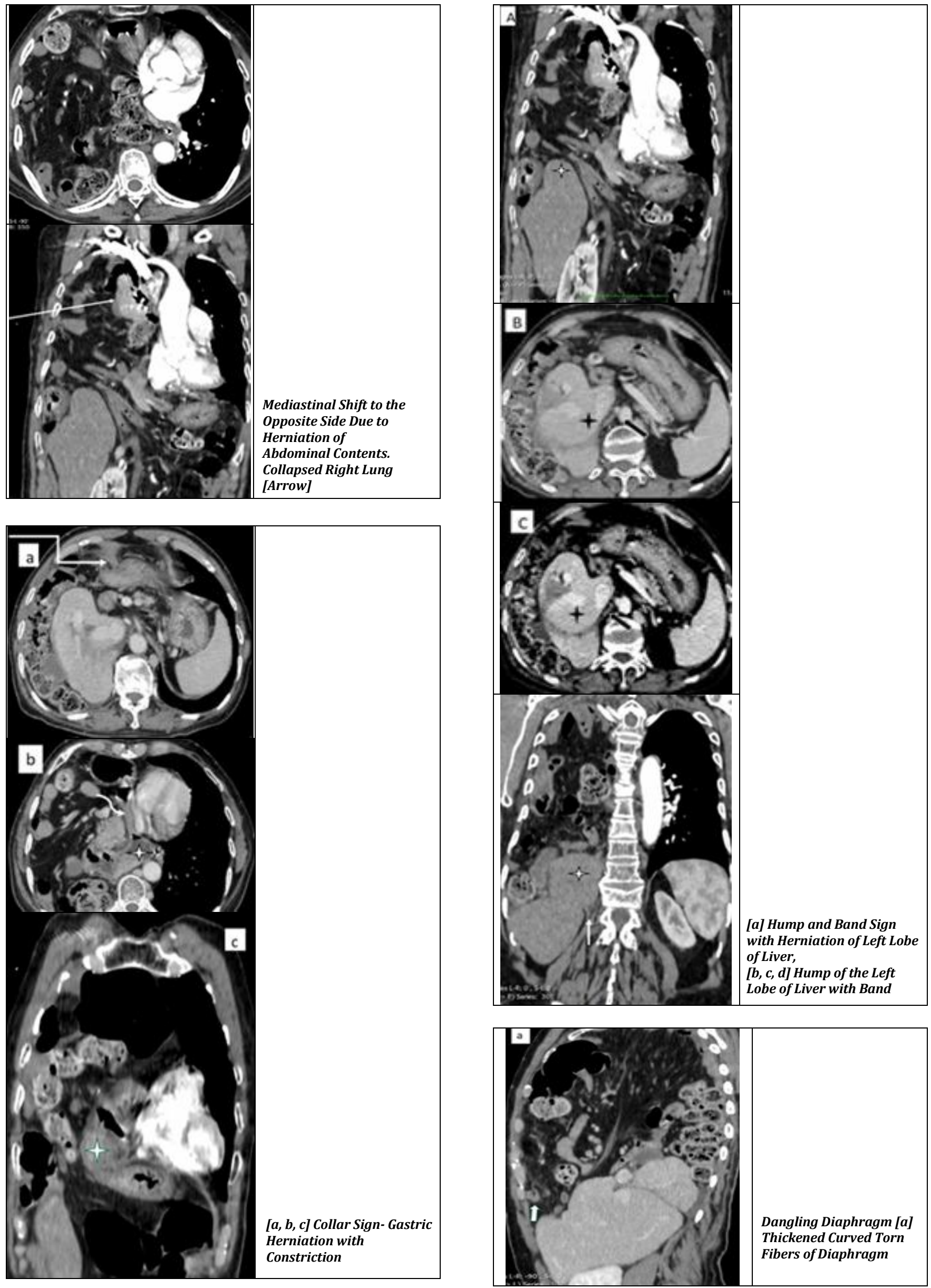


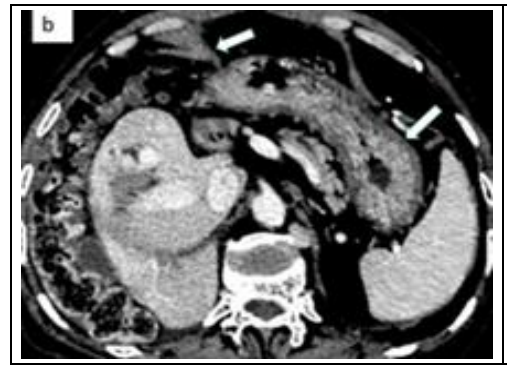

Dangling Diaphragm [b] There is Gastric wall Thickening (Arrow)

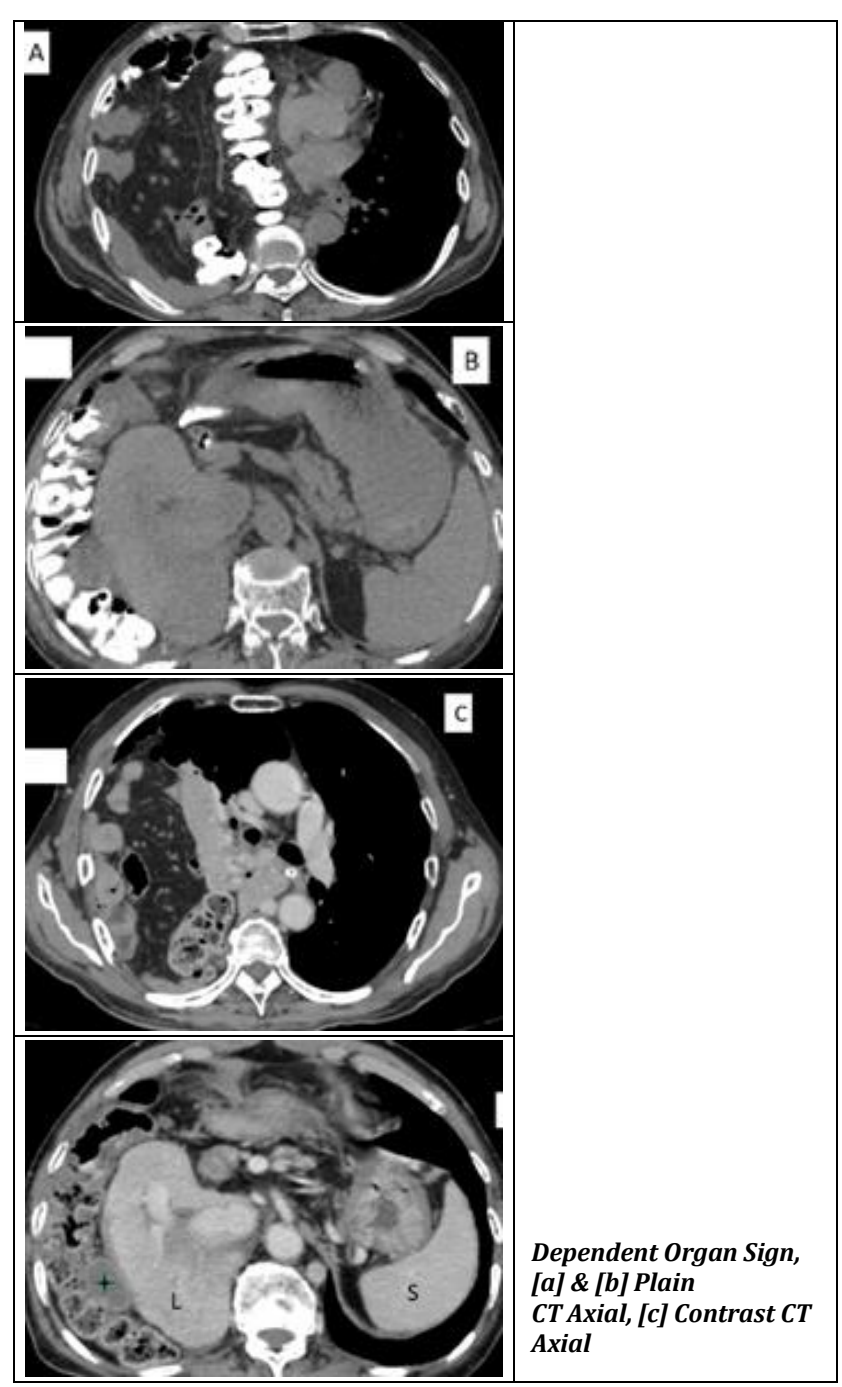

DISCUSSION

A congenital or acquired defect in the diaphragm causes herniation of intra-abdominal contents into the thoracic cavity. The most common cause of acquired diaphragmatic hernia is due to trauma.[1] In $3 \%$ of cases of abdominal injuries, the rupture of diaphragm causes thoracic herniation of intraabdominal contents.[4,6] Delayed presentation of diaphragmatic hernias occurs in $30 \%$. This is because most hernias are small in the early stages, but the size increases with passage of time due to herniation caused by thoracic and abdominal cavity pressure differences. ${ }^{[2]}$ The force vectors that develop during the impact, change the pattern of diaphragmatic rupture. Lateral impacts cause an anteroposterior elongation of the thoracic cage with subsequent rupture of the diaphragm or detachment of its insertions, whereas there is an abrupt increase in the intraabdominal pressure in the frontal impacts, transmitting the impact to the pillars of the diaphragm and its rupture. Rib fractures or injuries can cause the rupture of the insertion of the diaphragmatic and transverse rupture of the dome of diaphragm in extreme cases.[5,6] Most frequent rupture of the diaphragm occurs in lateral impacts and it usually tears towards the side of the impact.[7]

In non-penetrating traumas, right side ruptures are less frequent [12-40\%] due to greater resistance of the right hemidiaphragm and the protection by the liver compared to tears on the left side [ 50 to $88 \%$ of cases].[5, 8] Diaphragmatic hernia following rupture passes through three phases: 1 . The time of trauma represents the Initial phase; 2 . The enlargement of the defect and/or herniation occurs in the delayed phase (asymptomatic); and 3. The patient presents with various complications of diaphragmatic hernia in the third phase which is referred to as the obstructive phase.[1] In our case patient presented after 14 years of trauma and he presented with gastritis due to reflux of duodenal contents, which are possibly secondary to an abnormal position of bowel loops. According to the literature, one can diagnose diaphragmatic hernia on plain chest radiography in $73 \%$ of the cases. The signs that help in diagnosing the condition are intrathoracic herniation of abdominal viscera, multiple airfluid levels in the thoracic cavity, marked elevation of hemidiaphragm, distortion of diaphragmatic margins, and contralateral mediastinal shift. These classical signs are seen with established herniation, however, tear without herniation in an acute setting is difficult. ${ }^{[9,10]}$ The chest Radiograph of our patient showed these classical signs.

In proven cases of acute or delayed presentation of diaphragmatic herniation, ultrasound will be useful. Ultrasound cannot be used to diagnose isolated tear of the diaphragm except defining absent diaphragm movements.[11] As chest Radiograph showed classical presentation, our patient had not undergone an ultrasound examination. The investigation of choice for diaphragmatic hernia is CT scan (sensitivity of 73\%). The CT signs which help in diagnosing the diaphragmatic hernia include discontinuation of the diaphragm, Collar sign, herniation of viscera, elevation of the hilum of the liver, hemi diaphragmatic elevation, and dependent viscera sign. ${ }^{[8]}$ The herniation of stomach, spleen, colon, small bowel and liver are most commonly noticed, which are seen in our case. Surgical management changes on the basis of the time of presentation. Laparotomy is done in acute settings. In delayed settings, for treatment of intrathoracic adhesions, a thoracotomy approach may be required. In stable patients, various treatment options include laparoscopy, thoracoscopy or video-assisted thoracoscopic surgery (VATS).[12]

In our case, the patient was not willing for surgical correction and we counselled the patient about the delayed complication. For small defects, a primary surgical repair is warranted. While a mesh may be used in cases of large defects. Morbidity and mortality risk associated with right-sided diaphragmatic hernia is high because of its rare nature and difficulty in diagnosing the condition, along with various associated complications. A correct initial diagnosis can be established by taking into consideration the presenting complaints along with past history, coupled with a thorough assessment with the involvement of the radiologists. 


\section{Consent}

For the purpose of publication of this manuscript and any accompanying images, written informed consent was taken from the patient.

\section{REFERENCES}

[1] Viallonga R, Pastor V, Alvarez L, Charco R, Armengol M, Navarro S. Right-side diaphragmatic rupture alter blunt trauma. An Unusual entity. World J EmergSurg 2011;6:3.

[2] Jadloweic CC, Sakorafas LU. Delayed presentation of traumatic right-sided diaphragmatic hernia after abdominoplasty. Case Rep Surg 2014;2014:949531.

[3] Ebrahimi G, Bloemers FW. A delayed traumatic right diaphragmatic hernia with hemothorax. J Surg Case Rep 2012;2012(1):1.

[4] Goi G, Callegaro D, Villa R, Moroni E, Bondurri A, Danelli P. Large bowel obstruction as a result of occult diaphragmatic hernia 11 years after injury. Ann Ital Chir 2012;83(5):425-8.

[5] Panicek DM, Benson CB, Gottlieb RH, et al. The diaphragm: anatomic, pathologic, and radiologic considerations. Radiographics. 1988;8:385-425.
[6] Sangster GP, González-Beicos A, Carbo AI, et al. Blunt traumatic injuries of the lung parenchyma, pleura, thoracic wall, and intrathoracic airways: multidetector computer tomography imaging findings. EmergRadiol. 2007;14:297-310.

[7] Chughtai T, Ali S, Sharkey P, et al. Update on managing diaphragmatic rupture in blunt trauma: a review of 208 consecutive cases. Can J Surg. 2009;52:177-81.

[8] Scharff JR, Naunheim KS. Traumatic diaphragmatic injuries. ThoracSurgClin. 2007;17:81-5.

[9] Eren S, Kantarci M, Okur A. Imaging of diaphragmatic rupture after trauma. ClinRadiol. 2006;61(6):467-77.33.

[10] Sliker CW. Imaging of diaphragm injuries. RadiolClin North Am. 2006;44(2):199-211.

[11] Blaivas M, Brannam L, Hawkins M, Lyon M, Sriram K. Bedside emergency ultrasonographic diagnosis of diaphragmatic rupture in blunt abdominal trauma. Am J Emerg Med. 2004;22(7):601-4.

[12] Qurrat Al Ain Atif, TanwirKhaliq. Traumatic right diaphragmatic hernia; A delayed presentation: Case report.JAyub Med Coll Abbottabad.2016;28(3). 